\title{
Tumeur chéloïde
}

\author{
Keloid Tumor
}

\section{Soumia Ayachi}

Service d'oncologie médicale, centre anti cancer de Batna, Algérie

\section{Correspondance à :}

Soumia AYACHI

soumia.ayachi@icloud.com

DOI:https://doi.org/10.48087/ BJMSci.2018.5131

Il s'agit d'un article en libre accès distribué selon les termes de la licence Creative Commons Attribution International License (CC BY 4.0), qui autorise une utilisation, une distribution et une reproduction sans restriction sur tout support ou format, à condition que l'auteur original et la revue soient dûment crédités.
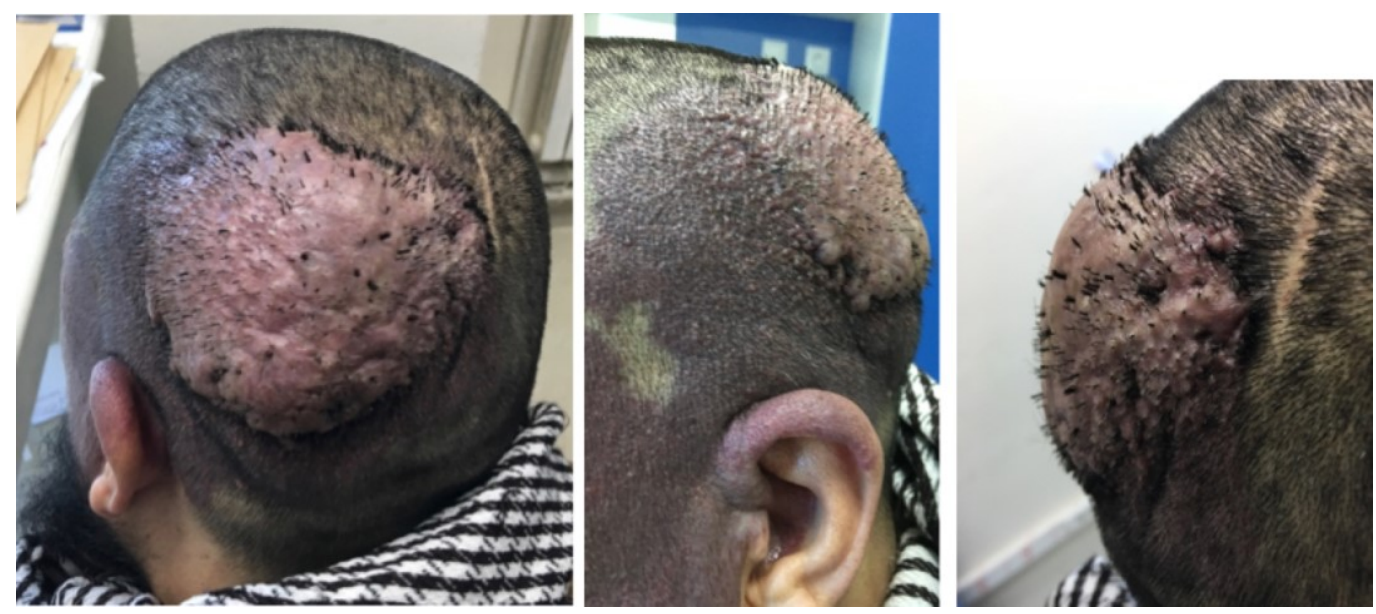

Un homme de 41 ans sans antécédents pathologiques qui présente une lésion éxophytique du scalpe au niveau occipito-temporal gauche mesurant $20 / 24 \mathrm{~cm}$ de grand axe a surface rugueuse évoluant depuis 2 ans avec un hémangiome congénital de la région faciale et temporale gauche.

Quel est le diagnostic le plus probable? Pathologie tumorale maligne ? Dermatoses granulomateuses (sarcoïdose, lèpre ...) ? Tumeur bénigne? Mycose cutanée éxophytique?

La bonne réponse est la 3ème vue la présence d'une lésion unique évoluant depuis 2 ans non ulcérée non surinfectée. L'examen anatomopathologique de la biopsie tumorale objectivait une tumeur chéloïde.

Les tumeurs chéloïdes sont des tumeurs fibro-prolifératives cutanées causées par une cicatrisation anormale de la peau lésée ou irritée par des traumatismes, brûlures, chirurgie, vaccination, piercing, piqure d'insecte, scarifications rituelles ou ethniques, tatouage, l'acné et l'herpès zostérien. Bien qu'ils soient des lésions bénignes, elles se développent dans la peau selon des modèles de croissance maligne. Ces tumeurs ont un impact majeur sur la qualité de vie des patients et elles représentent le cauchemar des jeunes filles surtout lorsqu'elles siègent au niveau des zones exposées du corps. Elles touchent jusqu'à $15 \%$ des populations à peau génétiquement pigmentée. Le mécanisme exact de pathogenèse est encore mal élucidé ; cependant, une altération du métabolisme de la matrice extracellulaire (un déséquilibre entre formation de dépôt de collagène et sa destruction) peut entraîner des cicatrices excessives

Elles prédominent au niveau de certaines topographies quelle que soit l'ethnie : les lobes des oreilles et les régions rétro-auriculaires, le cou, les faces externes des épaules, les omoplates, le thorax en zone pré-sternale. Elles sont le plus souvent prurigineuses et le grattage constitue certainement un facteur aggravant et probablement un élément prédictif du caractère évolutif.

Le diagnostic anatomopathologique est habituellement facile; on observe une prolifération conjonctive faite des faisceaux de collagène épais éosinophiles (hyalinisants) appelés " collagène chéloïdien » séparés par des faisceaux de fibroblastes, de l'œdème et des cellules inflammatoires sans réseau élastique; situé dans la couche réticulaire séparé de l'épiderme. Il ne faut pas les confondre avec d'autre tumeurs bénignes (histiocytofibrome, angiomyofibrome, fibrochondrome, cylindrome...) ou malignes (dermatofibrosarcome, maladie de Kaposi) ni avec des dermatoses granulomateuses (sarcoïdose, lèpre...) ou bien encore des mycoses exotiques.

Le traitement est toujours local ; il repose sur la chirurgie d'exérèse tumorale suivi de radiothérapie et application local de corticoïdes. Le profil évolutif des tumeurs chéloïdes est marqué par un risque important de récidive locale qui est de $23 \%$, mais jamais de dégénérescence. Les patients doivent consulter en urgence, au moindre signe d'activité : prurit, tension, augmentation de volume.

Déclaration d'intérêts : l'auteur ne déclare aucun conflit d'intérêt en rapport avec cet article. 